\title{
45. PHYSICAL PROCESSES IN COMETARY ATMOSPHERES
}

\author{
A. Z. DOLGINOV \\ A. F. Ioffe Institute of Physics and Technology, Leningrad, U.S.S.R.
}

\begin{abstract}
Formulae are obtained for the distribution of molecules in the cometary head, taking into account the conditions of hydrodynamic and free molecular flow in various regions around the nucleus. Experimental data are used to derive physical parameters near the nuclei of comets 1952 III, $1955 \mathrm{~V}, 1957$ III, and 1960 II and the rate of decrease of mass. The possibility of chemical reactions in the region close to the nucleus is discussed. Gas condensation is shown to be a possible cause of dust formation under the conditions existing near the nucleus, and this process may be responsible for the major portion of the cometary dust component. The observed grouping of synchrones in the cometary tail can be explained on the assumption that the nuclear surface comprises two (or more) areas differing essentially in evaporation rate, the amount of matter ejected varying over the rotation period of the nucleus. Charged dust particles are shown to form, with electrons and ions, a common medium, i.e., dust plasma, which can be treated by the same methods used for ordinary plasma. Special investigations appear to be desirable when comets intersect meteor streams.
\end{abstract}

The nongravitational forces acting on a cometary nucleus depend upon the evaporation rate of the constituent matter and on possible ejections from the nucleus. For studying these phenomena, it is essential to know the characteristics of the cometary atmosphere, primarily those of its gaseous and dust components, which are directly dependent upon the processes taking place in the vicinity of the nucleus.

\section{Neutral Gaseous Component of the Cometary Head}

The distribution function of neutral molecules can be found (Dolginov and Gnedin, 1966; Dolginov et al., 1970) from the kinetic equation

$$
\frac{\partial N}{\partial t}+\mathbf{v} \frac{\partial N}{\partial \mathbf{r}}+\mathbf{g} \frac{\partial N}{\partial \mathbf{v}}=S-\frac{N}{\tau}
$$

where $N$ is the number of molecules with velocity $\mathbf{v}$ at the point $\mathbf{r}$ at time $t, \tau$ is the lifetime of molecules prior to conversion to some other particles in the process of dissociation or ionization, $\mathbf{g}$ is the acceleration of the molecules due to light pressure and solar attraction, and $S$ is the number of particles emitted by the source per unit time.

We are not in a position to consider directly the processes occurring in the region near the nucleus as we have no direct data on the constituent substances of the nucleus. None of the observed molecules, such as $\mathrm{C}_{2}, \mathrm{CN}$ and $\mathrm{OH}$, is original; rather, they are formed in the neighbourhood of the nucleus as a result of the decay of primary particles owing to the Sun's electromagnetic and corpuscular radiation or to chemical conversion processes. However, compared to the size of the head, the region where particle collisions are important and where formation of the observed molecules takes place is small $(\leq 1000 \mathrm{~km})$, and this justifies our viewing the neigh- 
bourhood of the nucleus as an effective point source of molecules. Suitable conditions are to be provided at the source boundaries to assure transition from continuous medium flow to free molecular flow. It has been shown by Hamel and Willis (1966) that molecular distribution at such a boundary is characterized by a common radial outflow velocity $\mathbf{u}$, and a certain longitudinal temperature $T$ in a reference system moving at the velocity $\mathbf{u}$. The farther from the source, the closer the motion is to a radial trajectory, so that the transverse temperature (characterizing the spread in velocities normal to the radius) falls off rapidly to zero while the longitudinal temperature tends to a constant value. Granted the above assumptions, the problem is reduced to solving Equation (1) with the source function given by

$$
\begin{aligned}
& S(\mathbf{r}, \mathbf{v}, t)=A G(t) \exp \left[-\alpha\left(\mathbf{v}-u \frac{\mathbf{r}}{r}\right)^{2}\right] \frac{\delta(r)}{r^{2}} \delta\left(\frac{\mathbf{r}}{r}-\frac{\mathbf{v}}{v}\right) \\
& A=\left(\frac{\alpha}{\pi}\right)^{3 / 2}\left\{\left(1+2 \alpha u^{2}\right)[1+\varphi(\sqrt{\alpha} u)]+2 u \sqrt{\frac{\alpha}{\pi}} \exp \left(-\alpha u^{2}\right)\right\}^{-1} .
\end{aligned}
$$

Here $\alpha=(m / 2 K T)=1 / v_{T}^{2}$ is the reciprocal of the square of the thermal velocity of the molecules $v_{T}, m$ is the molecule mass, $G$ is the source power, i.e., the total number of particles (of all velocities) emitted by the source per unit time, $\delta(r)$ and $\delta\left(\frac{\mathbf{r}}{\tau}-\frac{\mathbf{v}}{v}\right)$ are Dirac's one-dimensional and two-dimensional delta functions, respectively, and $\varphi(x)$ is the error function.

Equation (1) with the source function described by Equation (2) has been solved (Dolginov et al., 1970). Only some basic results will be mentioned here. The distribution function is very dependent upon the Mach number $M=u / v_{T}$. Within the regions close to the nucleus $(\alpha g r \leqslant 1)$ but outside the regions of molecular collisions, we shall have, for $M \gg 1$,

$$
N(\mathbf{r}, t)=\frac{\sqrt{\pi} M G}{\alpha r^{2}}\left[1+\frac{\alpha \mathbf{g r}}{2 \sqrt{\pi} M} \exp \left(-M^{2}\right)\right],
$$

and for $M \ll 1$,

$$
N(\mathbf{r}, t)=\frac{G}{2 \alpha r^{2}}(1+\sqrt{\pi} M)[1+(1-\sqrt{\pi} M) \alpha \mathbf{g r}] .
$$

It can be seen that at small distances from the nucleus the hydrodynamic outflow velocity leads to reduced anisotropy in the density distribution, and at $M \gg 1$ the distribution is practically isotropic. At large distances, where $\alpha g r \gg 1$, we shall have

$$
\begin{aligned}
N(\mathbf{r}, t)= & \frac{G}{2 r} \sqrt{\frac{\pi g}{2 \alpha r}} \exp \left[-M^{2}-\alpha g r(1-\cos \psi)\right. \\
& \left.-\frac{1}{\tau} \sqrt{\frac{2 r}{g}}+2 M \sqrt{\alpha g r(1-\cos \psi)}\right],
\end{aligned}
$$

where $\psi$ is the angle between the radius vector of the molecule from the cometary nucleus and the prolongation of the radius vector of the comet from the Sun. 
From Equation (5) it follows that when $M \ll 1$ the particle concentration at the periphery of the sunward portion of the cometary head falls off exponentially. This concentration distribution is due to light pressure, which forces the molecules back into the tail. In the case where $M \gg 1$, at $r<2 M^{2} / \alpha g$ the fall-off in concentration is much slower on the sunward side of the head than on the opposite side, while at $r>2 M^{2} / \alpha g$ the position is reversed. The fact is that at high hydrodynamic pressure the particles accumulate in the sunward part of the head, having no time to migrate towards the tail. On the side away from the Sun, the effects of hydrodynamic velocity and light pressure augment each other. It is therefore possible that the concentration will be higher on the sunward side than on the back side. This was in fact observed for periodic comet Encke and comet 1965 VIII (Ikeya-Seki). Normally, however, the distribution is drawn away from the Sun, which indicates a low $M$ value.

The flux of light energy over a frequency range $\Delta v$ reaching a terrestrial observer from a sold angle $\Delta \Omega$ of the visible surface section of the coma and falling on a unit area normal to the line of vision is

$$
\begin{aligned}
I(t) & =B_{v}(t) \int_{0}^{\infty} \mathrm{d} \mathbf{r}_{p} \int_{\Delta \Omega} \mathrm{d} \Omega N\left(\mathbf{r}_{p}-\mathbf{r}_{Y,}, t\right) \\
B_{v}(t) & =c \int_{\Delta v} \mathrm{~d} \nu h \nu n_{v} \sigma_{v}(\vartheta, \varphi),
\end{aligned}
$$

where $n_{v}$ is the number of quanta of the solar spectrum at frequency $v$ per unit frequency range and per unit volume in the observed region of the comet, $\sigma_{v}(\vartheta, \varphi)$ is the cross-section of the re-emission at frequency $\nu$ of the quantum by the molecule per unit solid angle in a direction with polar coordinates $\vartheta, \varphi$ from the incident radiation, $c$ is the velocity of light, $h$ is Planck's constant, $\mathbf{r}_{N}$ is the vector from the observer on the Earth to the cometary nucleus, $\mathbf{r}_{p}$ the vector to the point of the coma under observation and $\mathbf{r}=\mathbf{r}_{p}-\mathbf{r}_{N}$.

The explicit form of $I(t)$, which can be obtained by substituting Equations (3) to (5) into (6) and by integration, is given by Dolginov et al. (1971). Comparison of the isophotes observed for various comets shows fairly good agreement with theory. Values of parameters characteristic of the physical condition of the coma and obtained by comparing theory with experiment are shown in Table I for various comets.

TABLE I

\begin{tabular}{|c|c|c|c|c|c|c|}
\hline Comet & $r(\mathbf{A U})$ & $\begin{array}{l}\text { Type of } \\
\text { molecule }\end{array}$ & $V\left(\mathrm{~cm} \mathrm{~s}^{-1}\right)$ & $T(\mathrm{deg})$ & $g\left(\mathrm{~cm} \mathrm{~s}^{-2}\right)$ & $\tau(\mathrm{s})$ \\
\hline $1955 \mathrm{~V}$ & 0.93 & $\mathrm{C}_{2}$ & $10^{5}$ & $1.7 \times 10^{3}$ & 0.395 & $1.35 \times 10^{5}$ \\
\hline 1957 III & 0.64 & $\mathrm{C}_{2}$ & $9.6 \times 10^{4}$ & $1.6 \times 10^{3}$ & 0.844 & $\begin{array}{l}\tau_{0}=3 \times 10^{6} \\
\tau_{1}=3.4 \times 10^{5}\end{array}$ \\
\hline 1960 II & 1.055 & $\mathrm{C}_{2}$ & $8.1 \times 10^{4}$ & $1.1 \times 10^{3}$ & 0.308 & $1.48 \times 10^{5}$ \\
\hline 1952 III & 1.2 & $C N$ & $1.2 \times 10^{5}$ & $2.5 \times 10^{3}$ & 0.365 & $2 \times 10^{5}$ \\
\hline 1957 III & 0.64 & $C N$ & $1.3 \times 10^{5}$ & $3.13 \times 10^{3}$ & 1.28 & $\begin{array}{l}\tau_{0}=1.8 \times 10^{5} \\
\tau_{1}=1.26 \times 10^{5}\end{array}$ \\
\hline
\end{tabular}

Characteristics of the comas of various comets 
It is noteworthy that in the case of comet 1957 III (Arend-Roland) two values, $\tau_{0}$ and $\tau_{1}$, are given for the lifetime of molecules in the solar radiation field. These have been calculated on the basis of isophotes observed on the sunward and rear positions, respectively. The difference can be explained on the assumption that the ultraviolet solar radiation responsible for dissociation and ionization is significantly absorbed by the coma molecules and dust particles. The results of processing the isophotes indicate that the lifetime of the molecules of this comet depends upon the angle $\psi$. To estimate the optical thickness of the coma, the lifetime of the $\mathrm{C}_{2}$ molecule in comet 1957 III may be compared with that of the same molecule in, say, comet 1960 II (Burnham). If both of these comets were optically thin, the lifetime ratio would be equal to the ratio of the squares of the heliocentric distances, i.e., to 0.36 in this particular instance. The observed ratio equals 20 . As the decrease in radiation intensity due to coma absorption is exponential, the ratio of these numbers gives $X_{1}-$ $X_{2}=4$, where $X_{1}$ and $X_{2}$ are the optical thicknesses of 1957 III and 1960 II, respectively. The ratio of $\mathrm{C}_{2}$-molecule lifetimes for comets $1955 \mathrm{~V}$ and $1960 \mathrm{II}$ is proportional to the ratio of the squares of the heliocentric distances, which is evidence that the comas of these comets are optically thin; i.e., $X_{2} \ll 1$ and hence $X_{1} \sim 4$.

It will be noted that it is not at all necessary for the optical thickness in the ultraviolet part of the spectrum to coincide with that of the visible region. Optical thickness may be used to obtain a lower estimate of the total rate $G$ of molecules leaving the source. For comet 1957 III $G \simeq 10^{30} \mathrm{~mol} \mathrm{~s}^{-1}$.

If Equation (6) is integrated over the total volume, an expression will be obtained for the absolute monochromatic brightness of the comet as a whole $I_{v}=B_{v} G \tau$. This expression is correct only for an optically thin comet, since $\tau$ is assumed to be constant throughout the head. Very few data are available on absolute monochromatic photometry of comets, and those available are usually inaccurate. Thus, $G$ is determined very poorly, but for comets of medium brightness it appears to be of the order of $10^{29}$ to $10^{30} \mathrm{~mol} \mathrm{~s}^{-1}$.

\section{Dust Component of the Cometary Atmosphere}

The usual explanation for the presence of dust in the cometary atmosphere is that the cometary nucleus is a conglomerate of various ices (such as $\mathrm{CH}_{4}$ and $\mathrm{NH}_{3}$ ) sublimating vigorously at relatively low temperatures and of contaminants in the form of dust particles of high melting-point. As the ices sublimate the dust particles are carried with the gas flows (Whipple, 1950).

An alternative mechanism (Dolginov, 1967) is that the dust particles form and grow due to gas condensation in the immediate proximity of the nucleus. As a matter of fact, some cometary gases, including such widespread gases as $\mathrm{C}_{2}$, are present in the coma in an oversaturated state and must condense into dust particles if a sufficient number of 'seeds' is present and sufficient collisions occur to enable the seeds to grow to dust-particle size. In the 'dirty' conditions surrounding the nucleus, where there are plenty of ions, as well as microscopic solid particles contained in gas, there will always be condensation centres in sufficient number, considering that the con- 
densing gases are not those which evaporate directly from the nucleus, but those formed as a result of dissociation or chemical reactions (e.g., $\mathrm{C}_{2}$ ). The concentration of condensing gases can be defined by the expression

$$
n(r)=\frac{Q}{v r^{2}}\left[1-\exp \left(\frac{R-r}{v \tau}\right)\right],
$$

where $Q$ is the number of primary particles formed per unit time, $v$ is the velocity of the primary particles, $\tau$ their lifetime, $R$ is the radius of the nucleus, and $r$ the distance from the centre of the nucleus to a given point. Particle growth is described by

$$
\mathrm{d} N_{g} / \mathrm{d} t=\beta n v S[1-\exp (-q \theta / T)], N_{g}=4 \pi \rho^{3} / 3 \delta,
$$

where $\delta$ is the number of atoms in a unit volume of dust-particle material, $N_{g}$ is the total number of atoms in a dust particle, $\rho$ the radius of the particle, $q=U / R$, where $U$ is the heat of sublimation and $\Re$ the gas constant, $\theta=\left(T_{H}-T\right) T_{H}^{-1}$ is the degree of oversaturation of the vapour ( $T$ being the vapour temperature and $T_{H}$ the vapour saturation temperature), $\beta$ is the coefficient of adherence of vapour molecules to dust particles (assumed to be close to unity), and $S=4 \pi \rho^{2}$.

For carbon in the vicinity of the nucleus $T<1000 \mathrm{~K}, T_{H} \simeq 2500 \mathrm{~K}, q \simeq 8 \times 10^{4} \mathrm{deg}$. The value of $\exp (-q \theta / T)$, determining the evaporation rate of the dust particles, is very small for the assumed values of $\theta, q$, and $T$ and can be omitted. Taking into account that $\mathrm{d} r=v \mathrm{~d} t$, we may derive from Equation (8) an expression for the increase in dust-particle radius with increasing distance from the nucleus:

$$
\frac{\mathrm{d} \rho}{\mathrm{d} r}=\frac{\beta Q}{v r^{2}}\left[1-\exp \left(\frac{R-r}{v \tau}\right)\right],
$$

the solution of which may be written

$$
\begin{array}{ll}
\rho=\beta \frac{Q \delta}{v R}\left[\frac{R}{v \tau} \ln \frac{v \tau}{R}-0.577 \frac{R}{v \tau}+\cdots\right] & R \ll v \tau \\
\rho=\beta \frac{Q \delta}{v R}\left[1-\frac{v \tau}{R}+\cdots\right] & R \gg v \tau .
\end{array}
$$

Further estimates are largely dependent upon the assumed lifetime $\tau_{p}$ of the primary molecules and density $\delta$ of the dust particles. Assuming $\delta=1.2 \times 10^{-23} \mathrm{~cm}^{-3}$ (graphite), $\beta=0.5, Q \simeq 10^{30} \mathrm{~mol} \mathrm{~s}^{-1}, v=10^{5} \mathrm{~cm} \mathrm{~s}^{-1}$ and $\tau_{p}=10$ to $100 \mathrm{~s}$, we obtain $\rho \simeq 10^{-4}$ to $10^{-5} \mathrm{~cm}$, which agrees perfectly with the observed dust-particle sizes.

The quantity of dust particles in the cometary atmosphere may increase either (1) due to the fact that a nuclear layer has been exposed, allowing it to evaporate and yield a large quantity of parent molecules for condensing matter, or (2) as a result of an increased intensity of hard electromagnetic or corpuscular solar radiation, leading to a greater yield from the chemical reactions that produce condensing substances. Hard radiations may be a decisive influence on the progress of reactions, even though they provide but a small contribution to the total radiation flux from the Sun. This may lead to a correlation between flares in cometary brightness and solar activity. 
A change in the chemical composition of the vapour towards increased quantity of condensing substances produces an increase in the brightness of the comet, even when the total amount of matter evaporating per unit time from unit surface area of the nucleus is constant. Increased brightness associated with condensation requires no additional energy; rather, it indicates liberation of energy. It is not necessary for outbursts in brightness to be associated with nuclear outbursts.

It should be emphasized that the conclusion regarding gas condensation into dust particles is unavoidable if the concentration of supersaturated vapours near the nucleus is $10^{12}$ to $10^{13} \mathrm{~cm}^{-3}$, which is in agreement with the existing estimates.

\section{Synchrones}

To be able to estimate the nongravitational effect upon the comet's trajectory, it is essential to know the relationship between the intensity of particle emission and the characteristics of the surface layer of the nucleus, and also whether the emission is a continuous process or a series of outbursts. We can acquire information about this by studying a system of synchrone clouds of dust particles simultaneously leaving the region near the nucleus. The very presence of separated synchrones is evidence of the discrete nature of ejections. Normally, synchrones emerge in groups, the time intervals between successive synchrone ejections being in many instances roughly equal. Thus, the successive tail-synchrone ejections from comet $1910 \mathrm{I}$ occurred on January $15.8,16.4,17.0,17.6$ and 17.9 , i.e., on the average, every 0.6 day. A similar pattern is observed with other comets having a system of synchrones. The most natural explanation appears to be that the nuclear surface consists of two areas which differ radically in the amount of matter emitted per unit time. If such a nucleus is in rotation, the total amount of matter emitted is modulated by the rotational frequency, and this results in a group of almost equally spaced synchrones. Certainly, the periodicity cannot persist for a long time, as a given nuclear layer may be totally exhausted, or a similar layer may be laid bare in another region, and so on. Since the synchrone formation occurs at the same phase of the rotation, the ejection will affect the comet's motion as a single reactive impulse. It would be interesting to analyse the fluctuations in brightness along the syndynames, even in the absence of pronounced synchrones. Such analysis can give important information about changes in the rate of evaporation from the nucleus and hence about the composition and rotation of the nucleus.

\section{Dust Plasma}

We shall not consider here the complicated problem of ion motion in type I tails. When considering the dust component of the cometary atmosphere, however, it is necessary to take into account the fact that dust particles take up a charge that depends on the dynamical equilibrium of the processes of electron absorption from the surrounding plasma and electron ejection by photons and fast particles. A common medium, which may be called a dust plasma, composed of ions, electrons, and charged dust particles that may be regarded as a special kind of heavy ions, is formed at 
distances sufficiently removed from the nucleus for the neutral particles to become ionized. Dust plasma will have collective properties characteristic of ordinary plasma. In addition to the waves characteristic of a collection of electrons and atomic or molecular ions there will be waves associated with the presence of charged dust particles. In order to determine the specific parameters of dust plasma it is necessary to know the distributions of mass and charge among the dust particles, but information on both distributions is very uncertain. The estimates normally quoted for a dust particle in the interplanetary medium at a distance of $1 \mathrm{AU}$ from the Sun lead to a positive charge of the order of 100 times that on an electron, in which case, a $10^{-5} \mathrm{~cm}$ particle will have a potential of $1.5 \mathrm{~V}$. The Debye radius in the cometary dust plasma is determined by the molecular ion concentration, which is much larger than that of dust particles.

Various types of dust-plasma oscillations are possible.

Comets are so large that they can be expected frequently to cross meteor streams. It would be interesting to pre-compute the dates of such crossings and observe the possible effects. Specifically, it would be of interest to establish whether the anomalous behaviour described by Biermann and Lüst (1965) for the plasma tail of comet 1964 VI (Tomita-Gerber-Honda) was due to its intersecting a meteor stream. To explain this phenomenon by interaction with an interplanetary magnetic field would require that the strength of the field was much greater than actually observed.

\section{References}

Biermann, L. and Lüst, Rh.: 1965, Mem. Soc. Roy. Sci. Liège Ser. 5 12, 329.

Dolginov, A. Z.: 1967, Astron. Zh. 44, 434.

Dolginov, A. Z. and Gnedin, Yu. N.: 1966, Icarus 5, 64.

Dolginov, A. Z., Gnedin, Yu. N., and Novikov, G. G.: 1971, Planetary Space Sci. 19, 143.

Hamel, B. B. and Willis, D. R.: 1966, Phys. Fluids 9, 829.

Whipple, F. L.: 1950, Astrophys. J. 111, 375.

\section{Discussion}

F. L. Whipple: Regarding condensation of dust near the comet, gas pressure should decrease with distance from the nucleus, and temperature should probably increase, contradicting the hypothesis. Also, dust and meteoroids must be of the same composition. Therefore iron, sodium, calcium, even hydrogen and many other elements must condense, not just carbon. Vaporization of these atoms in a comet nucleus seems unlikely.

A. $Z$. Dolginov: The absolute values of pressure and temperature, rather than their relative changes, are of importance for condensation. In principle, possible condensation processes for other elements may also be considered.

F. L. Whipple: I accept correction to my criticism if one allows possible growth of dust particles to dimensions of the order of microns.

G. Guigay: I should like to emphasize that synchrones are not always oriented in a radial direction; it was not the case for comet $1957 \mathrm{~V}$ (Mrkos), for example. Hence, the case you presented cannot be considered typical.

$A$. $Z$. Dolginov: The deflection of the synchrones from a radial direction is usually not large. I attempt here to explain only the large-scale properties of synchrones. The periodicity in the appearance of synchrones shows that the explanation is correct. To explain the deflection one must consider the development of the tail in more detail and take into account that the constituent matter of the synchrones is the dust plasma. 